\title{
Operation of Inertial Electrostatic Confinement Fusion (IECF) Device Using Different Gases
}

\author{
Gamal M. Elaragi ${ }^{1}$ \\ Published online: 19 January 2018 \\ (C) The Author(s) 2018. This article is an open access publication
}

\begin{abstract}
An optical signal of IEC plasma using different gases has been registered by means of an optical fiber and photomultiplier (PMT). The light passing through the fiber is directed to the entrance window of a photomultiplier (PMT, Hamamatsu R955), connected to the digital scope. The discharge current of plasma discharge has been recorded using current probe. The X-ray emission in IEC plasma device was investigated by employing time-resolved detector.
\end{abstract}

Keywords IEC $\cdot$ Discharge current $\cdot$ Plasma discharge $\cdot$ X-ray

\section{Introduction}

Inertial electrostatic confinement (IEC) is considered to be a promising future technology for space applications especially for advanced space propulsion systems. Since this technology was mainly investigated and developed for applications as neutron source until a few years ago, a great potential and need for investigations regarding discharge phenomena such as the propellant confinement and the jet extraction for thrust generation is still existent.

Unfortunately this principle suffers from many inherent loss mechanisms, which lead to a quick loss of ions at a small timescale and prevent a sufficient fusion gain $[1,2]$. Over the two last decades IEC devices have been mainly developed in two directions: The first one is the use as economical neutron sources with applications such as medical isotope production [3, 4] or baggage screening [5]. Other approaches have been taken into the direction of the development of a non-fusion plasma jet source for space propulsion [6, 7]. However, IEC devices have as well been proposed as a fusion energy source for future spaceships $[2,8,9]$. Most of these devices use a setup with a smaller grid nested inside a larger grid. These are usually spherical wire grids that feature a high transparency, but the

Gamal M. Elaragi

elaragi@gmail.com

1 Plasma Physics and Nuclear Fusion Department, Nuclear Research Center, EAEA, Cairo, Egypt openings between the wires have an unregularly shape and position. The average lifetime of ions in the plasma should be as long as possible [2].

One of the principal considerations while building a grid is to improve the grid transparency. Operation in the star mode depends more on the effective transparency versus the geometric transparency. However, operation in other modes is very dependent on the geometric value, so improvements in the geometric transparency are very desirable [10]. The material of grids for high power IEC devices has to withstand temperatures of up to $2000 \mathrm{~K}$ because ion bombardment can lead to rapid heating and material sputtering. For low power test devices, stainless steel provides an easier alternative for manufacturing. Novel approach for improving the neutralization in ioninjected IEC devices involves the active injection and confinement of electrons to the center of the device, to create dense, neutralizing plasma in the core region. Enhanced neutralization allows for higher ion densities and populations, and consequently higher fusion rates [11].

\section{Experimental Set-Up}

The IEC consists of a cylindrical vacuum chamber made of Pyrex glass tube with $10 \mathrm{~cm}$ of diameter and $30 \mathrm{~cm}$ height. The inner electrode consist of two stainless steel rings which are welded in their joint places (Fig. 1) and spiral outer electrode made of stainless steel. Figure 1 displays 


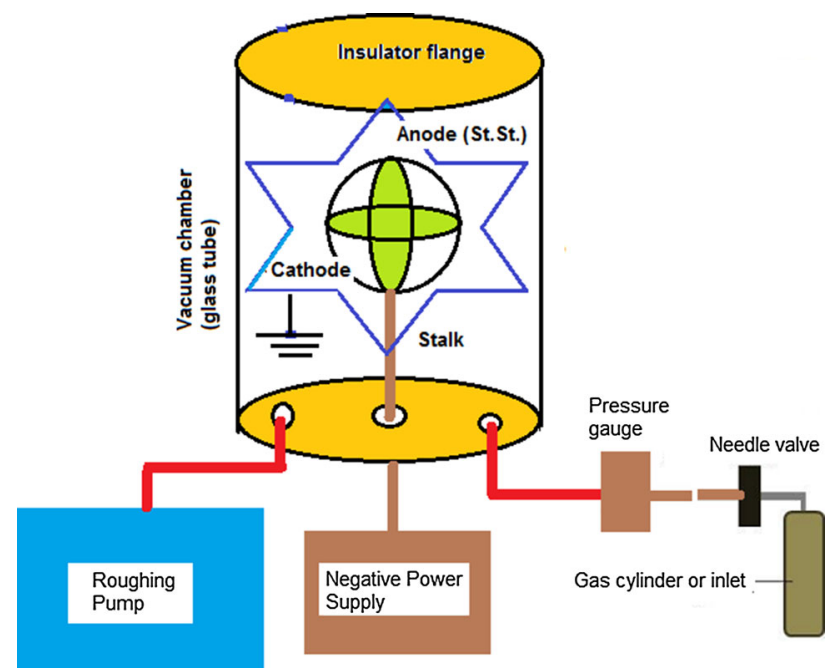

Fig. 1 Scheme of experimental setup

schematic diagram of the construction details of the IEC fusion set-up. Table 1 shows some parameters of IEC fusion device.

The discharges using the cathode with low effective transparency behave as solid cathode discharges. Thus, the discharge characteristics are primarily a function of the effective, rather than the geometric transparency of the cathode. For many IEC operations, a long grid lifetime is desirable, and this implies minimizing grid sputtering, not only by material selection but with choice of plasma operational modes such as using Star mode to minimize ion bombardment. The vacuum system consists of a rotary pump (Edwards single stage model 1 Sc.-150B). To avoid vapor from back streaming, the vacuum chamber is washed by gas after evacuation by the rotary pump. The gas was fed into the system via a flow meter in OMEGA model. The pressure was measured using a thermocouple gauge in Edwards's model.

Table 1 Design and operational parameters of IEC fusion device

\begin{tabular}{ll}
\hline Parameter & Value \\
\hline Vacuum chamber diameter & $10 \mathrm{~cm}$ \\
Vacuum chamber height & $30 \mathrm{~cm}$ \\
Anode grid diameter & $6.5 \mathrm{~cm}$ \\
Cathode grid diameter & $2.8 \mathrm{~cm}$ \\
High-voltage stalk height & $12 \mathrm{~cm}$ \\
Cathode voltage & $20 \mathrm{kV}$ \\
Gas pressure & $0.001-0.01$ Torr \\
\hline
\end{tabular}

\section{Power Supply Design}

D-D fusion can occur in an IEC device at voltages as little as $10 \mathrm{kV}$ or less, but detectable fusion generally does not occur until voltages are at least $15 \mathrm{kV}$ [11]. Preassembled power supplies are available but expensive. For this project, a negative DC power supply was built. A power supply, transformer $20 \mathrm{kV}$ and high voltage diodes have been used for this purpose. During the first positive halfcycle, which is designated on the sine wave graph as $\mathrm{T} 1$, the voltage from the transformer increases accordingly with the polarity shown. The current flows in the direction of the arrows, charging the capacitor through the diode. During the capacitor charging time there is no voltage to the inner grid because the current takes the course of least resistance. In other words, rather than take a path through ground and up to the plate of the cathode, the current swings up through the diode. The voltage across the capacitor will rise to the transformer secondary voltage to the maximum $20 \mathrm{kV}$ volts. As the transformer secondary voltage begins to decrease from its maximum positive value (at time increment $\mathrm{T} 2$ on the sine wave graph), the capacitor will attempt to discharge back through the diode. The diode is like a one-way street in that it will not conduct in this direction. Thus, the discharge path is blocked, and the capacitor remains charged to the $20 \mathrm{kV}$ volts.

At time T3, the transformer secondary (output) voltage swings into the negative half-cycle and increases in a negative direction to a negative $20 \mathrm{kV}$ volt. The transformer secondary and the charged capacitor are now essentially two energy sources in series. The $20 \mathrm{kV}$ volts across the transformer winding add to the $20 \mathrm{kV}$ volts stored in the capacitor and the sum voltage of $40 \mathrm{kV}$ volts is applied to the cathode. There are two fundamental characteristics of this $40 \mathrm{kV}$-volt output that should be noted. First, because a voltage doubler is also a rectifier, the output is a DC voltage. Second, the resulting output voltage that is applied to the http://www.micro techfactoryservice.com/magnetron.htmlcathode is actually a pulsed DC voltage. This is because the doubler generates an output only during the negative half-cycle of the transformer's output (secondary) voltage. So, the inner grid is, in fact, pulsed on and off at a rate of 50 times per second. Thus the voltage doubler circuit has been used to conduct the spark gap (S.G.).

Pulsed power supply circuit has allowed the inertial electrostatic confinement (IEC) fusion device to pulse to high currents, $170 \mathrm{~mA}$, at a peak voltage of $30 \mathrm{kV}$, and a pulse width of 0.016 microseconds. These results represent an increase in cathode currents several times of magnitude over steady state sources and enhancement the neutron production at the peak of the pulse (Fig. 2). 
Fig. 2 Circuit diagram of the power supply
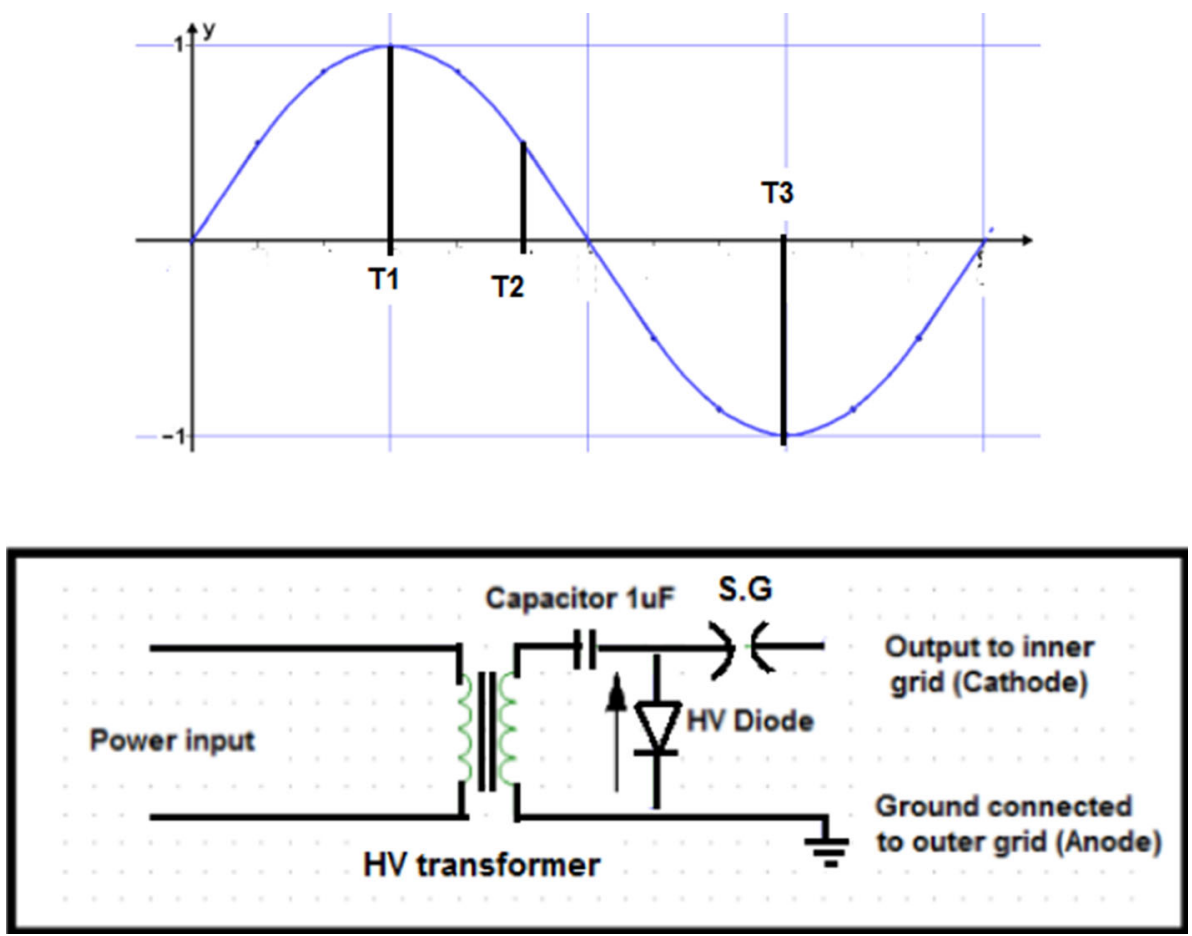

Figure 3 shows the relation between discharge current, in $\mathrm{mA}$ versus the discharge voltage, in $\mathrm{kV}$ using air gas. It is clear that the discharge current increases by increasing the discharge voltage. At higher voltages more electrons are extracted from the cathode surface per unit area and the current increases.

\section{Results and Discussion}

The discharge current through the discharge chamber was measured using a current monitor, which can be located upon returning to the ground. The signals from the current

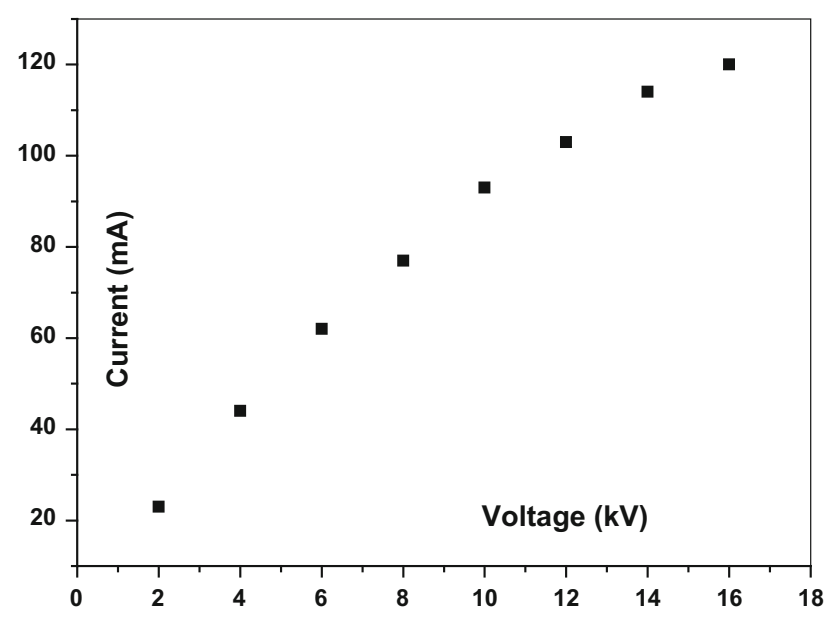

Fig. 3 Plot the relation between discharge voltage and discharge current for IEC plasma device monitor were recorded in a digitizing oscilloscope (Lecroy, USA) with a 200-MHz bandwidth. The light emitted by the plasma in the observation region was collected with an optical fiber and guided to a photomultiplier. A Hamamatsu photomultiplier tube (PMT) was used to detect coincident optical response. This PMT was mounted above and offset to the side of the plasma source, at a distance of $5 \mathrm{~cm}$ from the plasma source and the output signal was measured and recorded directly by a digitizing oscilloscope. A typical photomultiplier signal corresponds to plasma structures in the reactor for different gases can be observed. The photomultiplier voltage traces includes one pulse of interest is due to the primary discharge. Both intensity and duration of the pulses are different. This can be related to the different kinetics of elementary processes in the plasma structures. Although these results are obtained by measuring the total light emission from the plasma in the observation region, they represent valuable data of the plasma dynamics of fusion reactor (Table 2). The change in the full width at half maximum (FWHM) and amplitude of each signal is due to the kinetic energy of particles entering and leaving the plasma source.

Figures 4, 5, 6, and 7 shows the glow discharge, discharge current signal, time integrated X-ray intensity and PMT signal of hydrogen plasma source.

The radiation is the result of electron or ion interaction with others particles in the plasma. Because of their velocity, electron interaction tends to dominate the collision excitation and ionization processes. It was shown that increasing the mass number of the gas (air) decreased the 
Table 2 Intensity and full width at half maximum (FWHM) of visible light

\begin{tabular}{llll}
\hline & Air & Helium & Hydrogen \\
\hline Visible light intensity (a.u.) & 2.27 & 3.1 & 1.29 \\
FWHM (mS) & 2.8 & 5.7 & 4.1 \\
\hline
\end{tabular}

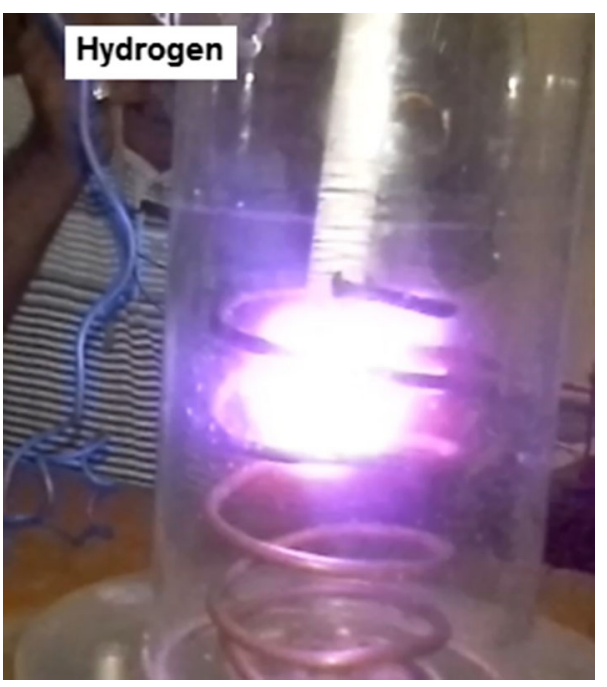

Fig. 4 Glow discharge of hydrogen plasma

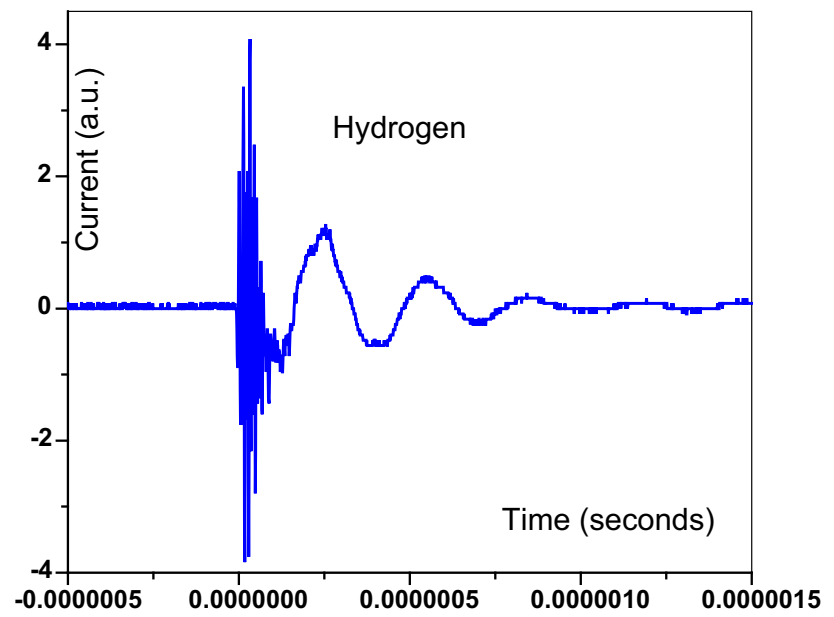

Fig. 5 Discharge current signal from hydrogen plasma

fluence, except that hydrogen had the lower values of the fluence energy and flux energy. Many gaseous phenomena depend on the $\mathrm{E} / \mathrm{N}$ variable, where $\mathrm{E}$ is the electric field and $\mathrm{N}$ is the number of molecules per $\mathrm{cm}^{3}$. E/N is often approximated by an $E / p$ variable, where $p$ is the gas pressure. The electron drift velocity is often expressed as a function of $E / p$, where $E$ is the electric field and $N$ is the number of molecules per $\mathrm{cm}^{3}$.

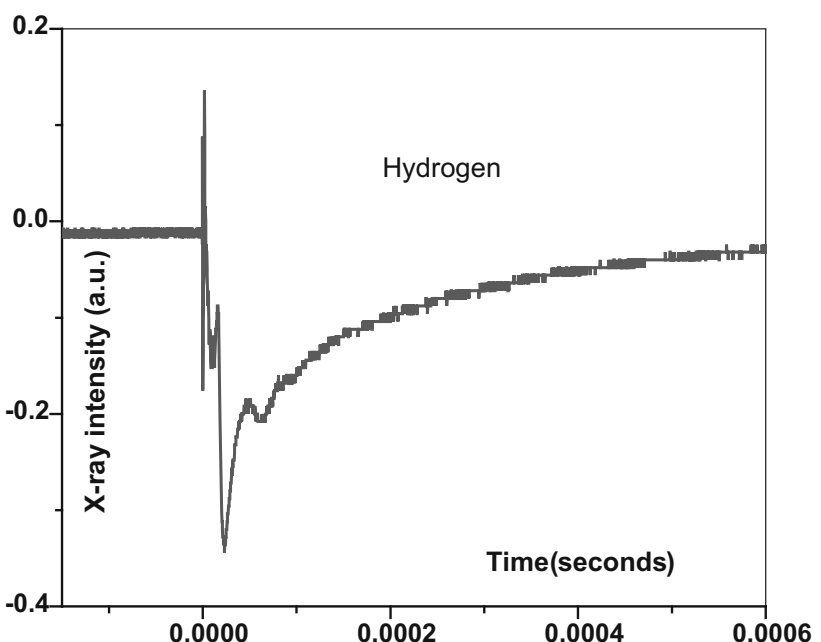

Fig. 6 Oscilloscope trace taken by PMT placed near from the IEC chamber

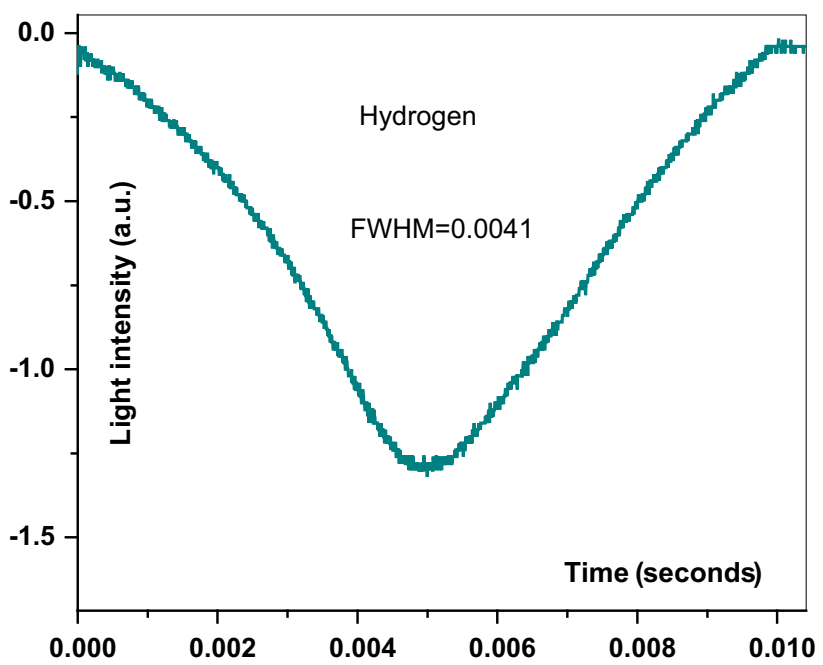

Fig. 7 PMT signal of hydrogen plasma source

Figures 8, 9, 10 and 11 shows the glow discharge, discharge current signal, time integrated X-ray intensity and PMT signal of air plasma source.

Figures 12, 13, 14 and 15 shows the glow discharge, discharge current signal, time integrated X-ray intensity and PMT signal of helium plasma source.

The generation of $\mathrm{X}$-ray has been investigated experimentally in the IEC fusion device using fast radiation detectors based on type of photomultiplier with high anode characteristics, nanosecond time resolution, shielded against electromagnetic interference in the assembly with fast scintillator. In the development of the detector housings special attention is paid to the screening of the pulsed electromagnetic interference occurring at the time of discharge. Table 3 indicates the relative X-ray intensity and FWHM of different gases (air, hydrogen and helium). 


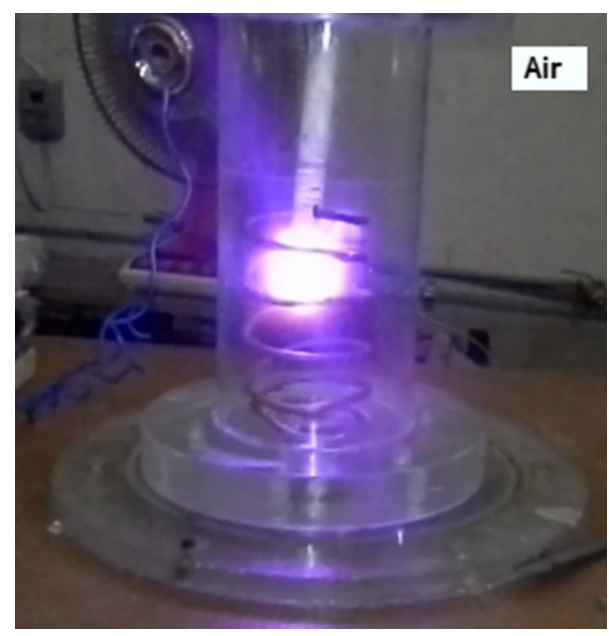

Fig. 8 Glow discharge of air plasma

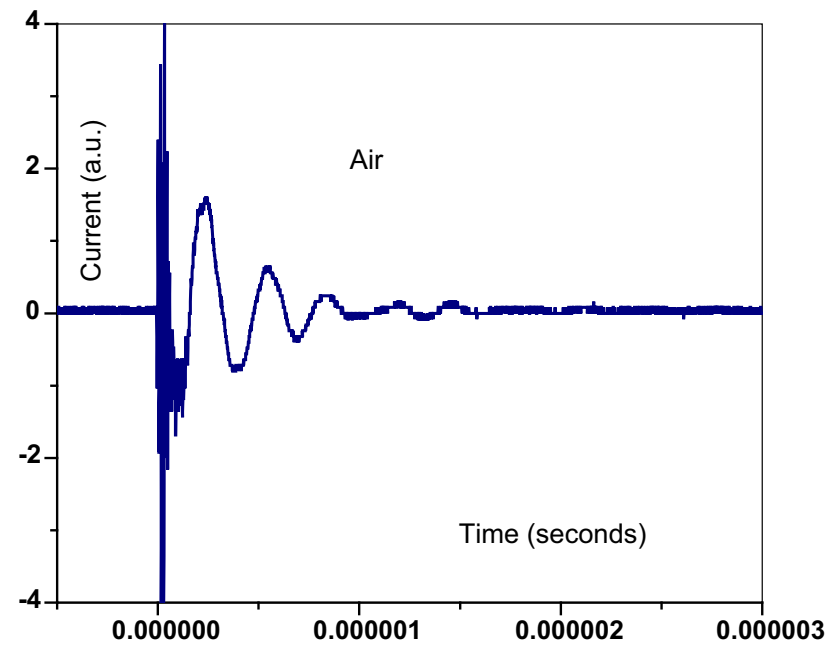

Fig. 9 Discharge current signal from air plasma

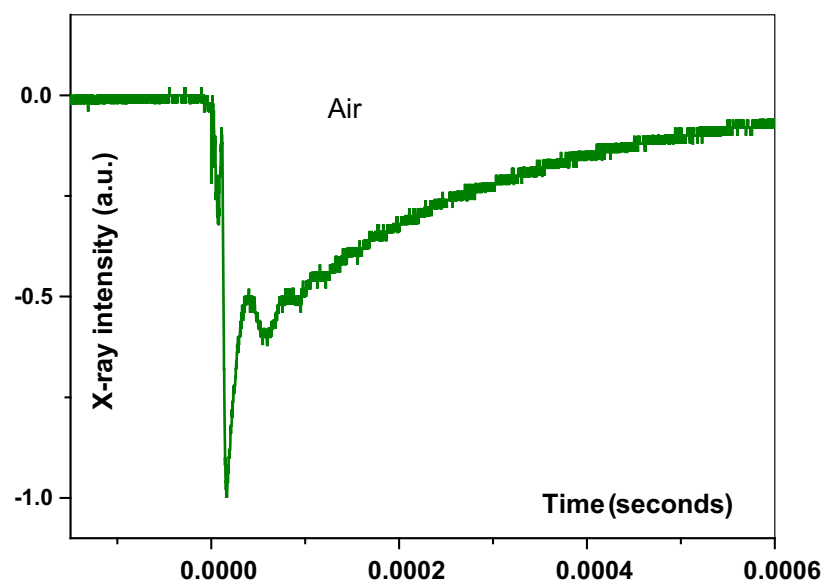

Fig. 10 Oscilloscope trace taken by PMT placed near from the IEC chamber

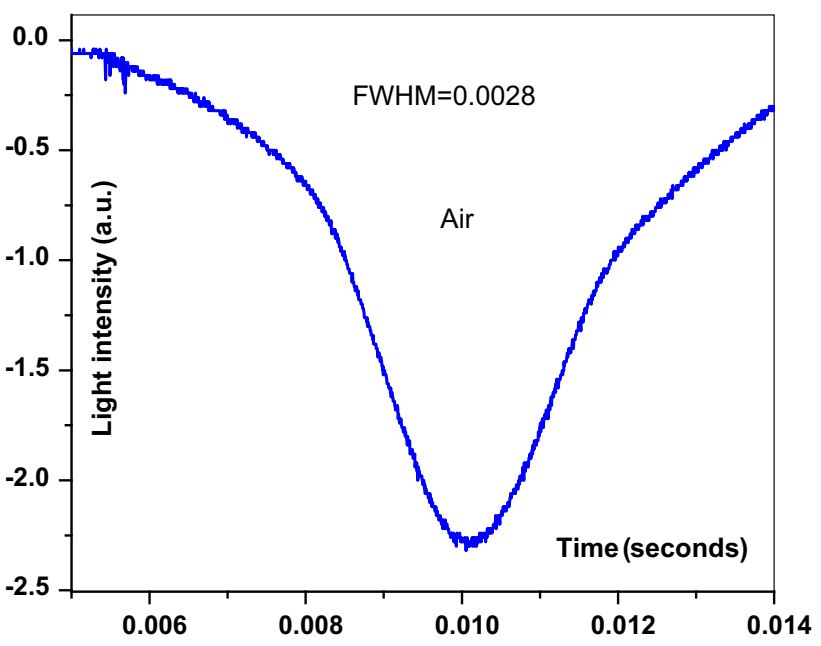

Fig. 11 PMT signal of air plasma Source

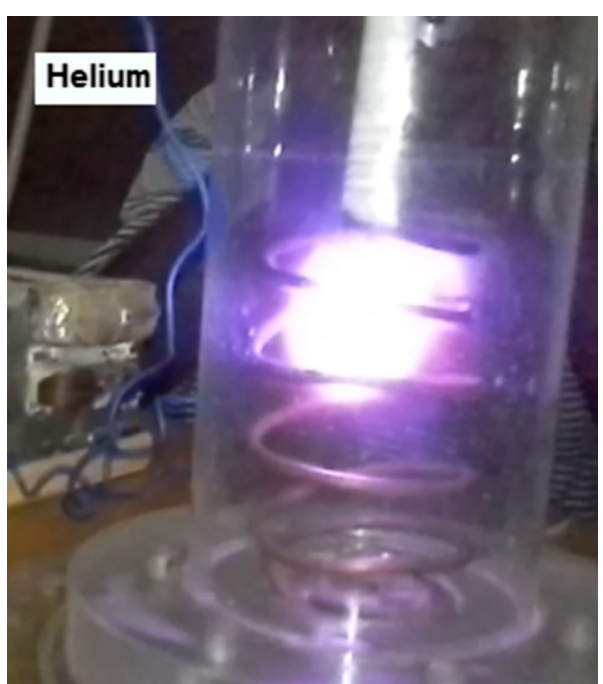

Fig. 12 Glow discharge of helium plasma

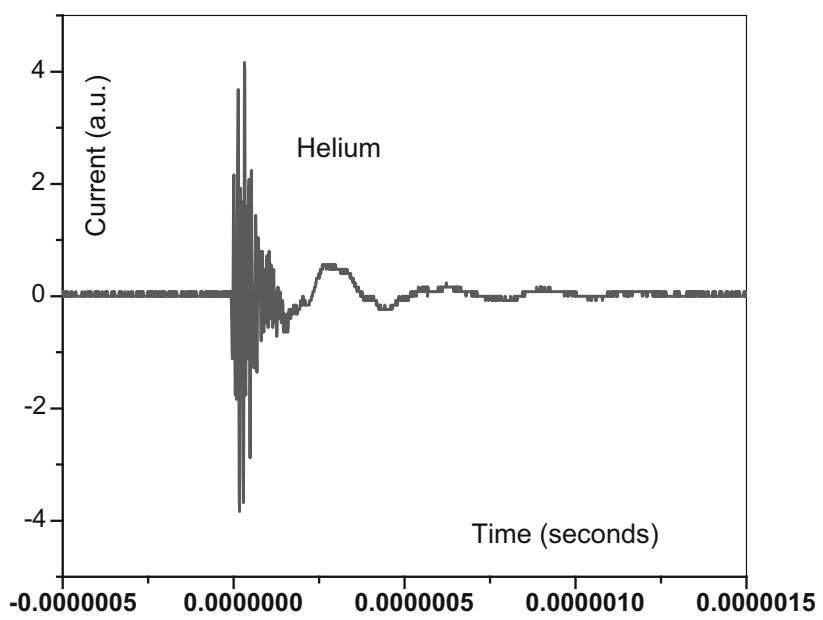

Fig. 13 Discharge current signal from helium plasma 


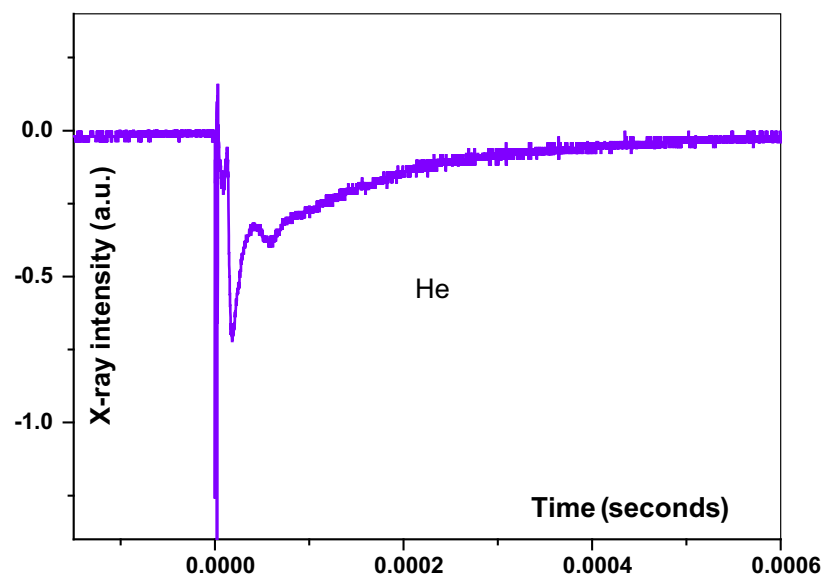

Fig. 14 Oscilloscope trace taken by PMT placed near from the IEC chamber

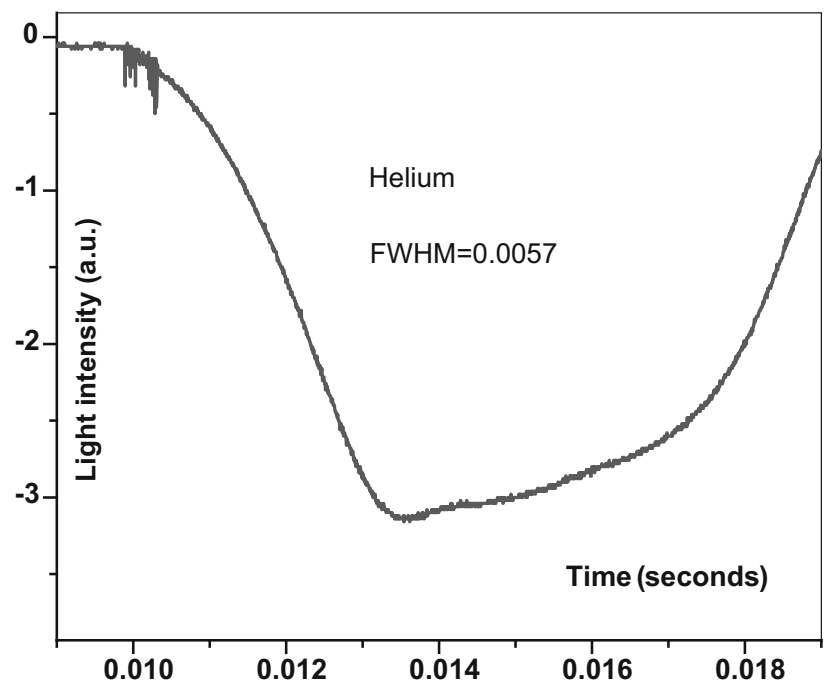

Fig. 15 PMT signal of helium plasma Source

Figure 16 indicates that the relation between X-ray intensity and the type of filling gas under operating conditions $(P=0.01$ Torr, $V \mathrm{ch}=20 \mathrm{kV})$. It was found that maximum intensity was registered for air gas when compared with other gases (helium and hydrogen).

Within a spherical containment vessel recirculatory focusing electrons are accelerated by a spherical grid within the vessel, and cause electron-electron collisions in a dense, central plasma core region of the sphere. The scattering interactions create intense bremsstrahlung $\mathrm{X}$ -

Table 3 X- ray intensity and full width at half maximum (FWHM)

\begin{tabular}{lrcc}
\hline & Air & Helium & Hydrogen \\
\hline X-ray intensity (a.u.) & 0.99 & 0.697 & 0.338 \\
FWHM $(\mu S)$ & 10.66 & 10.7 & 10.44 \\
\hline
\end{tabular}

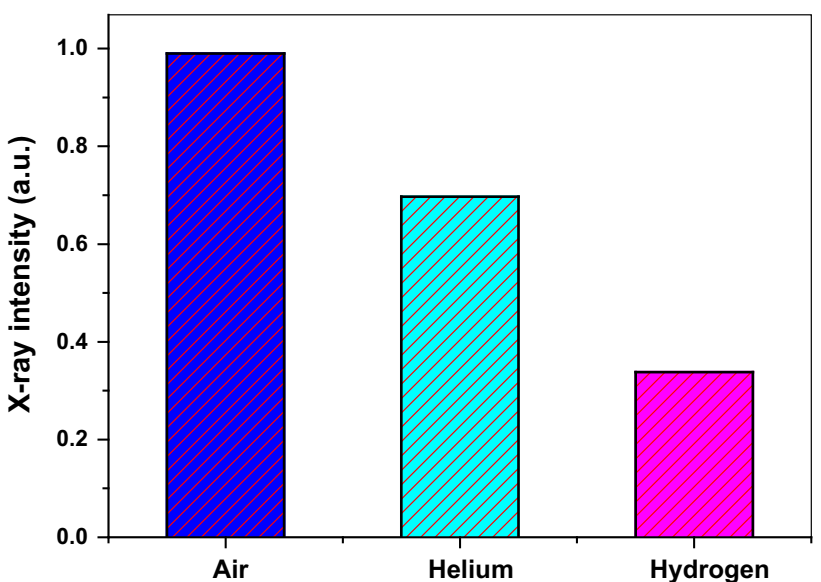

Fig. 16 Relation between X-ray intensity and type of filling gas

rays. The emitted X-ray energy is controlled by the grid bias. The potential for bremsstrahlung creation increases with increasing particle energy as well as increasing atomic number $(Z)$ of the absorber. The fraction of electron energy from a mono-energetic electron source, such as an X-ray tube, that is converted to photons is

$f_{e}=10^{-3} Z E_{e}$

where $E_{\mathrm{e}}$ is the electron energy in $\mathrm{MeV}$.

$\mathrm{X}$-ray generation with the IECF machines, continuum includes recombination and Bremsstrahlung radiation. The latter is caused by the accelerating electrons in the Coulomb field of ions, producing soft and hard X-rays. The interaction of the electron beam with anode also generates hard X-rays with energies up to $1 \mathrm{MeV}$.

Areas that have a higher optical intensity can be associated with an increase in energy and frequency of ion collisions, which can produce visible light [12]. The configuration with the best confinement of D-D plasma will be used in future experiments.

The confinement time can be restated in terms of the number of ion recirculations in the IEC potential well by dividing the well diameter by the average velocity of the recirculating ion. Work on reactor grade confinement for an IEC is closely tied to formation of a deep potential well and trapping of ions in that well sufficiently long enough (i.e., sufficient confinement time) to meet the Lawson Criteria. For a rough order of magnitude, assume that the converged region average density is around $10^{16} \mathrm{~cm}^{-3}$, then the confinement time needed is roughly $10 \mathrm{~ms}$. Radiation losses also need attention. 


\section{Fusion Fuel}

Deuterium makes a good fusion fuel because is easily obtained and because it has a cross section for fusion reactions that makes it obtainable within IEC fusion plasma. Figure 17 indicate the color of deuterium glow plasma. The typical time-resolved neutron detector signal is shown in Fig. 18.

The production of neutrons in an IEC plasma device is based on two main mechanisms: the thermal mechanism and the beam target mechanism. The thermal mechanism of neutron production is based on the collision of energetic deuterium ions inside the bulk of the plasma. The beam target mechanism in IEC plasma devices is caused by the interaction of accelerated deuterons with the plasma or background gas. The greatest number of fusion reactions is occurring inside and around the cathode-grid; and in most devices operating today, the majority of reactions is beambackground in nature, that is, between the fast moving ions in the plasma and the neutral background gas.

For using deuterium gas, the rate of DD-neutron generation is given by [13]

$\frac{d N}{d t}=\frac{1}{2} n_{i}^{2} \sigma\left(\epsilon_{i}\right) U_{i} \Omega$

where $\Omega$ is volume corresponding to maximum ion density, $\sigma$ is cross-section of fusion reaction, $n_{i}$ is the final density of deuterium ions and $U_{i}$ is the speed of deuterium ion.

The final density of deuterium ions is given by

$n_{i} \approx 2.7 \times 10^{2} f \varphi^{1.5} v^{2} \mathrm{~cm}^{-3}$

where $\varphi$ is the depth of potential well (the potential of the electric field in the axis of the system), $f \leq 1$ is the ratio of ion density to electron density and $v$ is the oscillation frequency is given by

$v \approx 8 \times 10^{6} \frac{\sqrt{\varphi}}{r_{g}} \mathrm{~Hz}$

where $r_{g}$ is the initial radius of inner cathode in $\mathrm{cm}$.

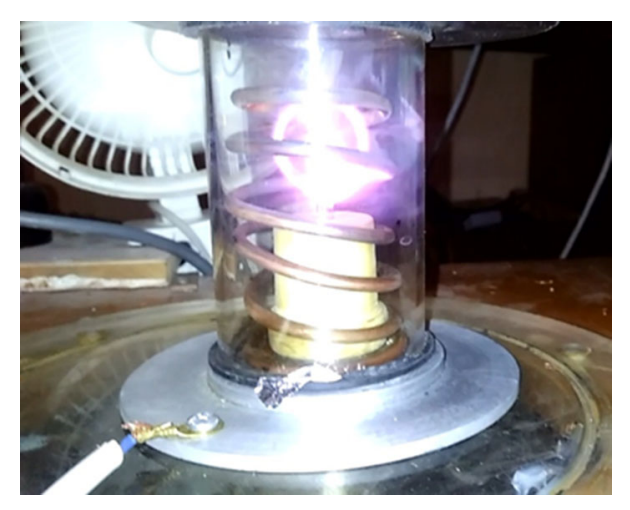

Fig. 17 Color of deuterium plasma

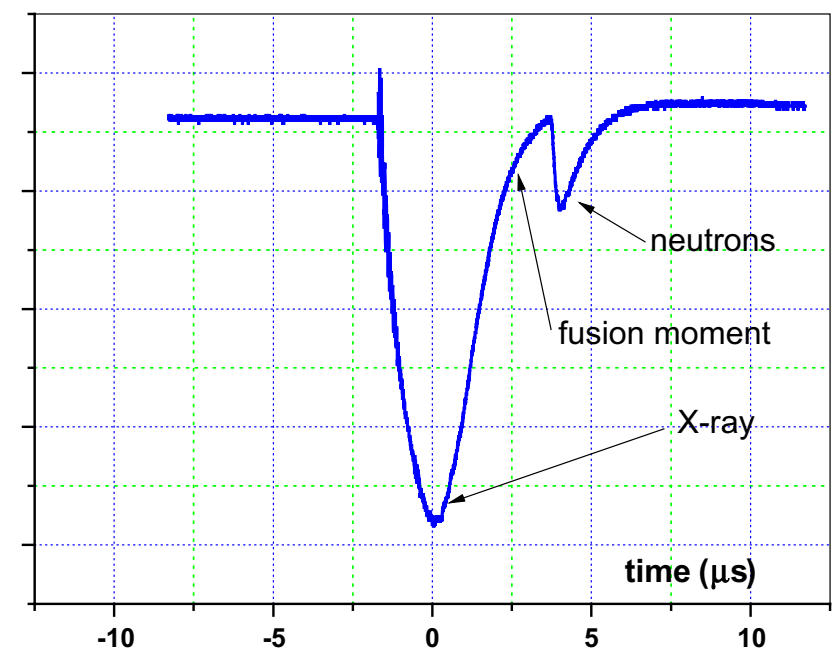

Fig. 18 Shows the typical time-resolved neutron detector signal

For $r_{\mathrm{g}}=1.4 \mathrm{~cm}, \varphi=20 \mathrm{kV}$ then the oscillation frequency equal $10 \mathrm{MHz}$ approximately and final density of deuterium ions equal $10^{16}-10^{18} \mathrm{~cm}^{-3}$ for $\varphi=20-40 \mathrm{kV}$.

Then the rate of DD-neutron generation (dN/dt) equal to $10^{6}-10^{8}$ neutrons/second.

\section{Conclusion}

According to the kinetic theory of gases, when gas pressure (or gas temperature) increases, the kinetic energy of gas increases, so it can reach to higher speeds. Therefore, more energetic particles emerge, as a result, more quanta of energy emerges in the form of bremsstrahlung radiations. The electrons directed toward anode are decelerated by the effect of the presence of target atoms of anode. These electrons lose their energy in the form of electromagnetic radiations. These phenomena are more responsible for the emission of hard X-rays. The scattering interactions create intense bremsstrahlung X-rays. The emitted X-ray energy is controlled by the grid bias. The calculated rate of DDneutron generation using the same electrode configuration about $10^{6}-10^{8}$ neutrons/second.

Open Access This article is distributed under the terms of the Creative Commons Attribution 4.0 International License (http://creative commons.org/licenses/by/4.0/), which permits unrestricted use, distribution, and reproduction in any medium, provided you give appropriate credit to the original author(s) and the source, provide a link to the Creative Commons license, and indicate if changes were made.

\section{References}

1. W.M. Nevins, Can inertial electrostatic confinement work beyond the ion-ion collisional time scale? Phys. Plasmas 2(10), 3804-3819 (1995) 
2. T.J. McGuire, Improved lifetimes and synchronization behavior in multi-grid inertial electrostatic confinement fusion devices. Ph.D. Dissertation, Aeronautics and Astronautics Department, MIT, Cambridge, MA, 2007

3. G. Miley, J. Sved, The IEC star-mode fusion neutron source for NAA — status and next-step designs. J. Appl. Radiat. Isot. 53, 779-783 (2000)

4. B.B. Cipiti, G.L. Kulcinski, Embedded D-He fusion reactions and medical isotope production in an inertial electrostatic confinement device. Fusion Sci. Technol. 44, 534-541 (2003)

5. A.L. Wehmeyer, The detection of explosives using an inertial electrostatic confinement DD fusion device. MS Thesis, University of Wisconsin, Madison, Wisconsin, 2005

6. G. Miley, Y. Gu, B.P. Bromley, J. Nadler, J. Sved, Plasma jet source using an inertial electrostatic confinement discharge plasma. Patent 6121569, 19 Sept 2000, USA

7. C. Syring, G. Herdrich, Experimental discharge characterization and scaling of IEC plasma devices, in IEPC-2013-289, Proceedings International Electric Propulsion Conference, Washington, DC, 2013

8. C.C. Dietrich, Improving particle confinement in inertial electrostatic confinement fusion for spacecraft power and propulsion.
Ph.D. Dissertation, Aeronautics and Astronautics Department, MIT, Cambridge, MA, 2007

9. R. Burton, H. Momota, N. Richardson, Y. Shaban, G.H. Miley, Fusion ship II-a fast manned interplanetary space vehicle using inertial electrostatic confinement fusion. AIP Conf. Proc. 654(1), 553-562 (2003). https://doi.org/10.1063/1.1541339

10. G.H. Miley, S.K. Murali, Inertial Electrostatic Confinement (IEC) Fusion Fundamentals and Applications (Springer, New York, 2014)

11. B. Sporer, A method for active space charge neutralization in an inertial electrostatic confinement (IEC) nuclear fusion on device. Ph.D. Dissertation, The Pennsylvania State University, Spring 2017

12. S. Krupakar Murali, B.B. Cipiti, J.F. Santarius, G.L. Kulcinski, Study of ion flow dynamics in an inertial electrostatic confinement (IEC) device through sequential grid construction. Phys. Plasmas 15, 122702 (2008)

13. S. Yu Gus'kov, YuK Kurilenkov, Neutron yield and Lawson criterion for plasma with inertial electrostatic confinement. J. Phys. Conf. Ser. 774, 012132 (2016) 